\title{
FOUR-COLOUR SURVEY OF W-UMA-TYPE SYSTEMS
}

\author{
S. M. R UCINS KI* \\ Warsaw University Observatory, Warsaw, Poland
}

\begin{abstract}
Four-colour photometry of $24 \mathrm{~W}$ UMa-type systems is presented.
\end{abstract}
Use has been made of the good separation of the spectral bands on both sides of the Balmer jump in Stromgren $u v b y$ photometry, to clarify the origin of the ultraviolet excesses $\delta(U-B)$ attributed by Eggen (1967) to the decreased metal abundance in some of the WUMa-type systems. 24 systems visible during the early spring months were observed with four to five observations contributing to the mean as listed in Table I. Contrary to expectations the $c_{1}$-index in the surveyed systems is rather normal for expected gravities whereas some systems indeed do show the decreased $m_{1}$-index leading to $\Delta m_{1}$ which well correlates with the $\delta(U-B)$ excess as given by Eggen. It can be

\section{TABLE I}

Four-colour photometry of W UMa-type systems

\begin{tabular}{lccccc}
\hline Name & Observed range in $V$ & $b-y$ & $m_{1}$ & $c_{1}$ & Remarks \\
\hline & & & & & \\
SS Ari & $10.1-10.5$ & +0.409 & +0.201 & +0.365 & \\
AH Aur & $10.2-10.5$ & +0.402 & +0.199 & +0.424 & \\
TZ Boo & $10.5-10.9$ & +0.421 & +0.133 & +0.279 & \\
XY Boo & $10.4-10.7$ & +0.331 & +0.131 & +0.369 & \\
AC Boo & $10.0-10.1$ & +0.390 & +0.158 & +0.305 & 2 obs. only \\
44i Boo & $4.7-4.8$ & +0.409 & +0.203 & +0.295 & with visual comp. \\
TX Cnc & $10.0-10.2$ & +0.392 & +0.200 & +0.352 & \\
RZ Com & $10.4-10.9$ & +0.354 & +0.174 & +0.297 & \\
CC Com & $11.3-12.0$ & +0.778 & +0.558 & +0.057 & changes in $c_{1}$ \\
BV Dra & $7.8-8.5$ & +0.367 & +0.143 & +0.308 & \\
BW Dra & $8.6-9.0$ & +0.422 & +0.150 & +0.317 & \\
YY Eri & $8.1-8.3$ & +0.419 & +0.203 & +0.319 & \\
AK Her & $8.4-8.7$ & +0.342 & +0.167 & +0.408 & with visual comp. \\
FG Hya & $10.0-10.2$ & +0.390 & +0.18 & +0.332 & \\
UZ Leo & $9.5-10.0$ & +0.242 & +0.143 & +0.683 & \\
XY Leo & $9.4-10.3$ & +0.561 & +0.359 & +0.341 & changes in $c_{1}$ \\
AM Leo & $9.1-9.3$ & +0.354 & +0.181 & +0.331 & with visual comp. \\
ER Ori & $9.3-9.5$ & +0.364 & +0.177 & +0.342 & \\
Y Sex & $9.8-10.1$ & +0.302 & +0.146 & +0.466 & \\
RZ Tau & $10.1-10.7$ & +0.422 & +0.071 & +0.610 & \\
W UMa & $7.7-8.3$ & +0.411 & +0.199 & +0.296 & \\
AW Uma & $6.8-7.1$ & +0.239 & +0.147 & +0.613 & \\
AG Vir & $8.4-8.5$ & +0.155 & +0.169 & +0.822 & \\
AH Vir & $9.1-9.7$ & +0.484 & +0.286 & +0.343 & \\
& & & & & \\
& & & & &
\end{tabular}

\footnotetext{
* Visiting Astronomer in Kitt Peak National Observatory which is operated by the Association of Universities for Research in Astronomy, Inc., under contract with the National Science Foundation. 
concluded that although rather large observed variations of the $c_{1}$-index in latest-type systems might indicate intrinsic emissions shortward of the Balmer jump, the origin of the $\delta(U-B)$ excesses should be attributed to the elevated flux level in the $v$-band of the four-colour photometry.

\section{Reference}

Eggen, O. J.: 1967, Mem. Roy. Astron. Soc. 70, 111. 